\title{
Time series analysis for water resources management - application to observed and simulated time-series of the groundwater flow numerical model of the coastal plain of Cecina
}

\section{Analisi delle serie temporali per la gestione delle risorse idriche - applicazione a serie temporali osservate e simulate dal modello numerico di flusso delle acque sotterranee della pianura costiera di Cecina}

\author{
Stefano Menichetti ${ }^{\mathrm{a}}$, Stefano Tessitore ${ }^{\mathrm{a}}$ \\ ${ }^{a} A R P A$ Toscana - Direzione Tecnica, via N. Porpora 22, 50144, Firenze, Italia \\ email: s.menichetti@arpat.toscana.it
}

\section{ARTICLE INFO}

Ricevuto/Received: 06 September 2021 Accettato/Accepted: 24 September 2021 Pubblicato online/Published online: 30 September 2021

Handling Editor: Rudy Rossetto

Citation:

Menichetti S, Tessitore S (2021) Time series analysis for water resources management - application to observed and simulated time-series of the groundwater flow numerical model of the coastal plain of Cecina Acque Sotterranee - Italian Journal of Groundwater, 10(3), 31 - 38 https://doi.org/10.7343/as-2021-531

Correspondence to: Stefano Menichetti s.menichetti@arpat.toscana.it

Keywords: MODFLOW, groundwater flow model, water budget, time series decomposition, net cumulative storage.

Parole chiave: MODFLOW, modello di flusso delle acque sotterranee, bilancio idrico, decomposizione serie temporali, immagazzinamento netto cumulato.

Copyright: (C) 2021 by the authors Licensee Associazione Acque Sotterranee This is an open access article under the CC BY-NC-ND license: http://creativecommons.org/licenses/bync-nd/4.0/

\section{Riassunto}

In questo lavoro sono evidenziate le potenzialità dell'analisi statistica delle serie temporali come strumento per la gestione del bilancio idrico delle acque sotterranee, mediante la tecnica di decomposizione applicata alla modellazione numerica di flusso in regime transitorio.

Sono presentati i principali risultati ottenuti applicando questa tecnica ad alcune serie temporali osservate ed a serie temporali derivate dal modello di flusso delle acque sotterranee della pianura costiera di Cecina (Regione Toscana, Italia), sviluppato in regime transitorio per il periodo 2005 - 2017.

Le serie storiche analizzate si riferiscono a precipitazioni, livelli idrometrici, livelli piezometrici ed esiti del bilancio, attraverso la derivazione della serie temporale dell'immagazzinamento netto cumulato. L'analisi di quest'ultima ha permesso, in particolare, di separare e quantificare due significative componenti del bilancio idrico: la riserva regolatrice $\operatorname{Wr}\left(22 \mathrm{Mm}^{3}\right)$ e la risorsa dinamica Wd $\left(2,5 \mathrm{Mm}^{3}\right)$. Il confronto di queste due componenti con i volumi di prelievo medio annuo da pozzi $\left(6,4 \mathrm{Mm}^{3} / y\right.$ nel periodo 2005 - 2017) ha fornito la misura di un bilancio idrico potenzialmente critico, soprattutto in periodi con ripetute tendenze climatiche avverse. Questo tipo di analisi, unita ad una modellazione "operativa", con aggiornamenti in tempo reale, è proposta in questa sede come strumento per la valutazione di possibili azioni preventive e di pianificazione della risorsa idrica sotterranea.

\section{Abstract}

This paper highlights the potentiality of the time series decomposition applied to transient regime groundwater flow models, as water balance management tool.

In particular, this work presents results obtained by applying statistical analysis to some observed time series and to time series derived from the groundwater flow model of the coastal plain of Cecina (Tuscany region, Italy), developed in transient regime within the period 2005-2017.

The time series of rainfall, river stage and bydraulic heads were firstly analysed, and then time series decomposition was applied to the "accumulated net storage", to finally discern and quantify two meaning ful components of the groundwater budget, the regulatory reserve $\left(W r=22 \mathrm{Mm}^{3}\right)$ and the seasonal resource $\left(W d=2.5 \mathrm{Mm}^{3}\right)$. These values compared with withdrawal volumes (average of $6.4 \mathrm{Mm}^{3} / y$ within the period 2005-2017) allowed to highlight potentially critical balance conditions, especially in periods with repeated negative climatic trends. Operational monitoring and modeling as following corrective and planning actions for the groundwater resource are suggested. 


\section{Introduzione}

Gli strumenti di modellazione attraverso la verifica diretta di ipotesi e concettualizzazioni permettono di raggiungere una conoscenza approfondita del complesso sistema delle acque sotterranee, così da supportare al meglio possibili misure per la loro protezione. La disponibilità odierna di serie temporali di dati idrogeologici sempre più estese consente, da un lato, di validare in modo sempre più accurato i risultati forniti dai modelli, dall'altro, attraverso numerose variabili riprodotte dal modello, di analizzarne la loro dinamica di funzionamento su periodi altrettanto estesi. Lanalisi statistica delle serie storiche dispone oggi di software di facile utilizzo che consentono una diffusa replica, anche in ambito applicativo, di analisi di estremo interesse già condotte in passato (Polemio and Dragone 1999). Il tema dell'analisi delle serie temporali riveste oggi un particolare interesse in ragione delle evidenze di cambiamento climatico e delle sue temute influenze sul mantenimento delle risorse idriche sotterranee con studi e comparazioni di varie tecniche di analisi (Meggiorin et al. 2021; Lee et al. 2018; Chen et al. 2021; Hasda et al 2021).
L'acquifero costiero della pianura di Cecina, la cui ubicazione geografica è riportata nella Figura 1, è stato oggetto di numerosi studi idrogeologici (Bartoletti et al. 1985) e di modellazione numerica per molteplici motivi di interesse, quali il bilancio idrico (Ambrosio and Fagioli 2001), la contaminazione da nitrati (Baldini et alii 2004), lo studio dell'intrusione salina (Genovese 2003) e le misure di contenimento di un grande pennacchio di contaminazione da composti organoalogenati (Armellini et al. 2015). Recentemente (ARPAT 2018) è stata realizzata una riunione di questi modelli di flusso in un unico modello su cui è stata effettuata una simulazione in regime transitorio per un periodo di 13 anni dal 2005 al 2017.

La realizzazione e i risultati del modello sono ampiamente descritti in uno specifico report di ARPAT (2018). In particolare dal bilancio idrico è stato derivato l'immagazzinamento cumulato (ARPAE 2010).

Il presente articolo si focalizza unicamente sulla presentazione e la discussione di possibili applicazioni operative, finalizzate alla gestione delle risorse idriche basate sull'analisi di questa voce di bilancio idrico, unitamente ed in comparazione con quella svolta sulle serie storiche di dati pluviometrici, idrometrici e freatimetrici.
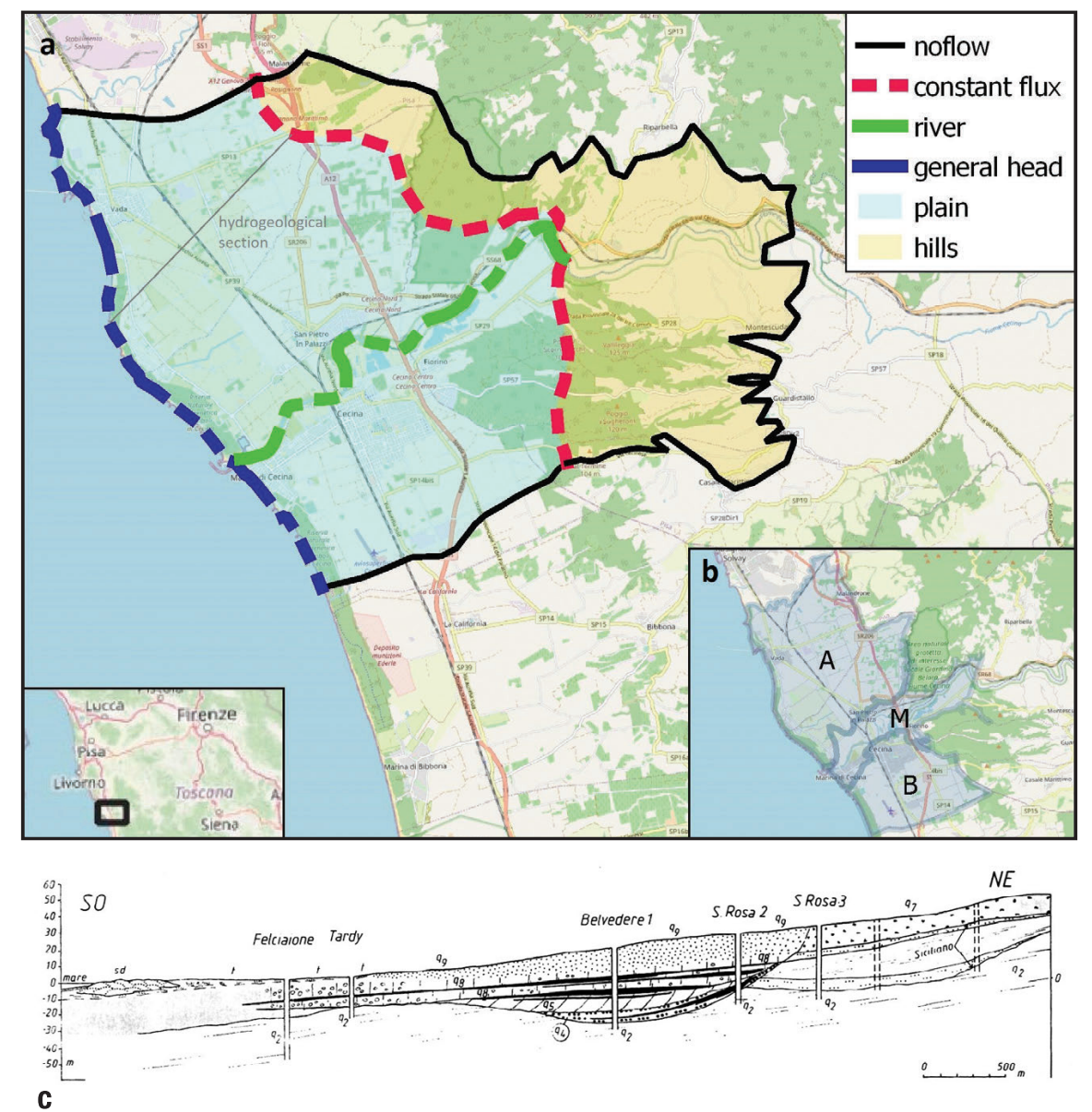

Fig. 1 - a) Geographical and hydrogeological setting of the study area and investigated model domain - (b) A, B, M water resource management zone. (c) Hydrogeological crosssection (Bartoletti et al., 1985): substrate Pleistocene aquiclude clays (q2), aquifers contained in the sandy, arenaceous and conglomeratic horizons (Pleistocene).

Fig. 1 - (a) Inquadramento geografico ed idrogeologico dell'area di studio e del modello analizzato. (b) Ambiti A, B ed M di gestione della risorsa idrica. c) Sezione idrogeologica (Bartoletti et al. 1985): substrato argille pleistoceniche acquicludo (q2), falde acquifere contenute negli orizzonti sabbiosi, arenacei e conglomeratici del pleistocene (q4, q5, q7, q9). 


\section{Materiali e metodi}

Lanalisi delle serie storiche rappresenta un metodo di indagine statistica atto a scomporre un qualsiasi processo sviluppato su base temporale in una parte deterministica ed una casuale residuale. Nell'analisi classica (Kendall and Stuart 1983), come implementata nel software R (Wuertz et al. 2017), la scomposizione in componenti stagionali, di tendenza e irregolari è realizzata tramite medie mobili con scelta tra modelli additivi o moltiplicativi. Lapplicazione determina e rimuove prima dalla serie temporale la componente di tendenza, utilizzando una media mobile con finestra simmetrica. In seguito, viene ottenuta la componente stagionale, calcolando la media per ciascuna unità di tempo, su tutti i periodi. Sottraendo dai dati osservati la componente di tendenza e quella stagionale si ottiene la componente finale di errore o residuale. Nel presente lavoro si è proceduto alla decomposizione sia di dati misurati e utilizzati come input del modello (precipitazioni, livelli idrometrici e piezometrici), sia dei dati dell'immagazzinamento netto cumulato, ottenuto dal bilancio idrico del modello in regime transitorio. Per tali operazioni di analisi statistica è stato utilizzato un modello di tipo additivo, generalmente considerato idoneo per dati di tipo idrologico.

Oltre la decomposizione della serie, si è proceduto ad un'analisi dell'autocorrelazione (Venables and Ripley 2002) della componente di tendenza, finalizzata all'individuazione di ciclicità pluriennali nei dati idrologici. In questo procedimento il coefficiente di correlazione lineare fra le variabili casuali $\mathrm{X}_{\mathrm{i}}$ e $\mathrm{X}_{\mathrm{i}+\mathrm{k}}$, al variare del numero intero $\mathrm{k}$, è definito sfasamento (lag). L'analisi dell'autocorrelazione è espressa da grafici denominati correlogrammi, che riportano la funzione di autocorrelazione (ACF) che consiste nei valori dei coefficienti di autocorrelazione, esplicitati per valori positivi di k. La funzione ACF mostra generalmente un andamento prima decrescente, a partire da valori prossimi all'unità, seguito da eventuali minimi o massimi. Il superamento da parte di un minimo o di un massimo dei limiti di confidenza riportati sul grafico determina il rifiuto dell'ipotesi nulla che non vi sia autocorrelazione, negativa o positiva, in corrispondenza del dato intervallo o lag. In sintesi la ciclicità è riconosciuta come non casuale e statisticamente significativa.

Risultano concettualmente analoghi i diagrammi di crosscorrelazione (Brockwell and Davis 1991) dove, in questo caso, il calcolo ripetuto del coefficiente di correlazione al variare del lag è condotto tra due variabili diverse e dà luogo alla funzione di cross-correlazione (CCF).

Il modello numerico idrogeologico preso in esame (ARPAT 2018) per l'applicazione delle tecniche di analisi delle serie temporali è stato elaborato in regime stazionario e transitorio con 52 stress periods trimestrali per 13 annualità, dal 2005 al 2017, utilizzando il codice USGS MODFLOW (Harbaugh and McDonald 1996) attraverso l'interfaccia software ESI GWVistas (Rumbaugh and Rumbagh 2007). Esso rappresenta l'unione di più modelli precedentemente implementati ed è suddiviso negli ambiti (A, B e M) di gestione della risorsa idrica, definiti dalla locale autorità di regolazione del Genio
Civile regionale nella pianura costiera di Cecina (Fig. 1).

Il modello rappresenta un area di pianura che si presenta come una fascia di dune a Sud-Ovest, che con più ordini di terrazzamenti marini (Fig. 1 - Bartoletti at el. 1985) si raccorda ai rilievi collinari a Nord - Est. Dal punto di vista geologico sono presenti depositi incoerenti quaternari del Pleistocene di origine continentale (fluviale ed eolica), marino-transizionale e marina. Nel complesso è stato ipotizzato un sistema multistrato a comportamento idrodinamico monofalda (Armellini et al. 2015), che si sviluppa tra la superficie del terreno, in buona parte sub-pianeggiante, ed un acquicludo inferiore costituito da uno spesso orizzonte argilloso, di età variabile dal Pliocene al Pleistocene inferiore. I dati misurati intervenuti nella modellazione ed analizzati con la tecnica delle serie temporali, sono quelli acquisiti fino al 31/12/2017 e riferiti alle stazioni indicate nella successiva Tabella 1.

Tab. 1 - Regional Hydrological and Geological Service Stations.

Tab. 1 - Stazioni Servizio Idrologico e Geologico Regionale.

\begin{tabular}{|l|c|l|l|c|}
\hline \multicolumn{1}{|c|}{ Station ID } & $\begin{array}{c}\text { Station } \\
\text { name }\end{array}$ & \multicolumn{2}{c|}{ Measure } & Period \\
\hline liv_TOS01005415 & Steccaia Q & h hydrometric & meters & $2009-2017$ \\
\hline liv_TOS19000601 & 5A & h piezometric & meters & $2004-2017$ \\
\hline prec_TOS11000002 & Cecina & rainfall & $\mathrm{mm}$ & $2012-2017$ \\
\hline
\end{tabular}

Sulla base dei dati di bilancio del modello è stato derivato l'idrogramma dell'immagazzinamento netto cumulato (ARPAE 2010) che descrive nel periodo considerato una oscillazione di tipo sinusoidale quasi completa, con un minimo assoluto nell'estate 2012 ed un massimo assoluto nella primavera 2016.

La curva di "immagazzinamento netto cumulato" è ottenuta sommando la differenza dei flussi in ingresso ed in uscita dalla componente di storage moltiplicati per $\mathrm{i}$ corrispondenti step temporali. La curva che rappresenta, di fatto, una funzione "serbatoio" per la misura delle variazioni di volume immagazzinato nel corpo idrico (Fig. 2) è stata oggetto dell'analisi della serie temporale

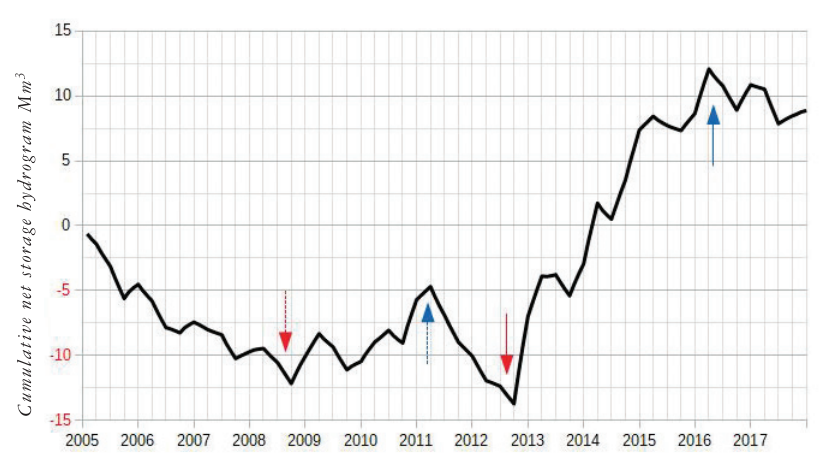

Fig. 2 - Cumulative net storage hydrogram $\mathrm{Mm}^{3}$.

Fig. 2 - Idrogramma dell'immagazzinamento netto cumulato $\mathrm{Mm}^{3}$. 


\section{Risultati e discussione}

Nella Figura 3 è riportata la decomposizione della serie temporale 2012-2017 del pluviometro di Cecina. La componente di tendenza mostra un ampio massimo tra 2014 e 2015 seguito da un trend discendente fino ai valori minimi del 2017. La stagionalità ha massimi autunnali (ottobre) e minimi tardo primaverili (giugno) ed estivi (agosto).

Decomposition of additive time series

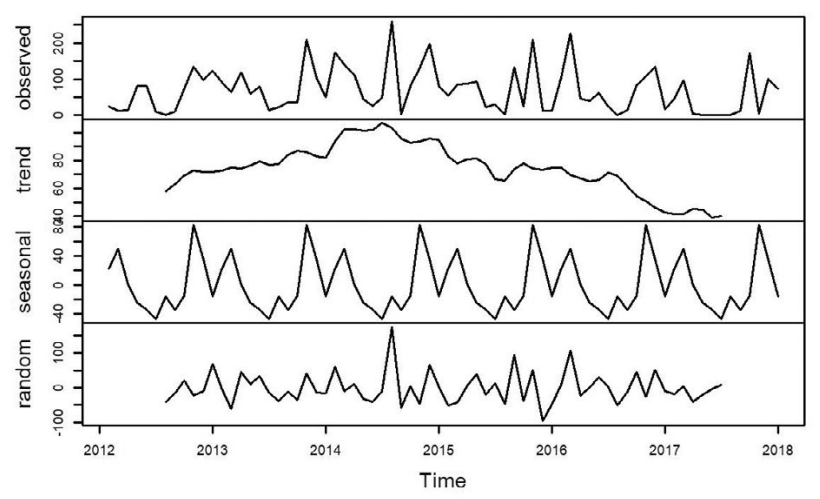

Fig. 3 - Decomposition of Cecina rain gauge time series.

Fig. 3 - Decomposizione serie temporale pluviometro Cecina.

La serie temporale 2009-2017 delle altezze idrometriche a Steccaia (Fig. 4) presenta un trend con cicli pluriennali e tendenza all'incremento, un massimo assoluto è stato raggiunto nel 2017, massimi relativi nel 2011 e 2014. Minimi assoluti a fine 2009 e nel 2012. La stagionalità ha massimi autunnali (novembre) e minimi tardo estivi (settembre).

Decomposition of additive time series

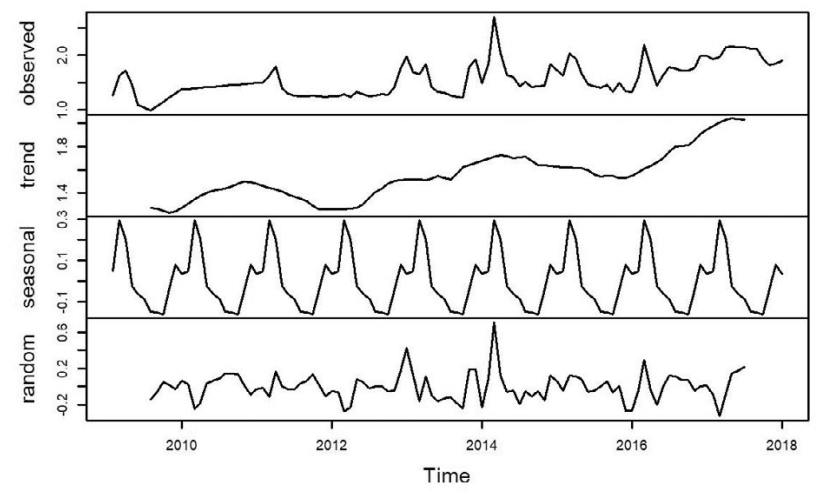

Fig. 4 - Decomposition of the Steccaia bydrometer time-series.

Fig. 4 - Decomposizione serie temporale idrometro Steccaia.
La serie temporale 2004-2017 delle altezze piezometriche sulla stazione freatimetrica $5 \mathrm{~A}$ (Fig. 5) mostra nella componente delle tendenze un generale incremento dal 2006 al 2011, un profondo minimo, nel 2012 come per la serie temporale precedente, nonché un successivo massimo nel 2014. La stagionalità ha massimi tardo invernali (marzo) e minimi tardo estivi (settembre).

Decomposition of additive time series

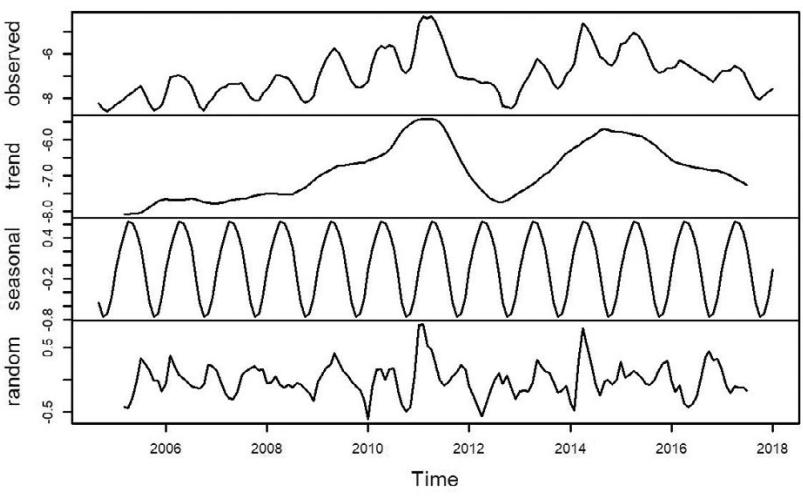

Fig. 5 - Decomposition of the 5 A groundwater level time-series.

Fig. 5 - Decomposizione serie temporale freatimetro 5A.

In sintesi, come riassunto nella Tabella 2 seguente, si osservano in generale periodicità pluriennali, con il 2011 e il 2014 come anni di massimo, separati dal 2012 come anno di magra.

Le stagionalità delle piogge e dei deflussi superficiali determinano massimi ripetuti nei mesi di ottobre, novembre e febbraio e minimi estivi in agosto. La risposta dei deflussi sotterranei è strettamente connessa a questa stagionalità, il minimo deflusso per esaurimento è ritardato di un mese (settembre), mentre la ricarica con i massimi piezometrici, per via della somma dei due massimi pluviometrici (autunno e inizio primavera) segue anche questa un ritardo di un mese (marzo)

Le analisi di autocorrelazione delle tre serie temporali, in particolare delle componenti di trend, confermano l'esistenza di periodicità pluriennali. I lags indicati nelle Figure 6 e 7 corrispondono ad un unità di anno solare, mentre le linee tratteggiate ad una soglia di significatività statistica. Un massimo della funzione di autocorrelazione ACF superiore alla soglia di significatività indica per il lag una periodicità completa (massimo - massimo), mentre un minimo la presenza perlomeno di un semiperiodo (massimo - minimo).

Tab. 2 - Results of the time-series decomposition study.

Tab. 2 - Risultati dello studio di decomposizione delle serie temporali.

\begin{tabular}{|c|c|c|c|c|c|c|}
\hline \multirow{2}{*}{ Station } & \multirow{2}{*}{ Measure } & \multirow{2}{*}{ Period } & \multicolumn{2}{|c|}{ Multi-year trend } & \multicolumn{2}{|c|}{ Seasonality } \\
\hline & & & $\max$ & $\min$ & $\max$ & $\min$ \\
\hline Steccaia Q & h hydrometric & $2009-2017$ & 201120142017 & 20092012 & November & September \\
\hline $5 \mathrm{~A}$ & h piezometric & $2004-2017$ & 2014 & 2012 & March & September \\
\hline Cecina & rainfall & $2012-2017$ & 20142015 & 2017 & October & June August \\
\hline
\end{tabular}




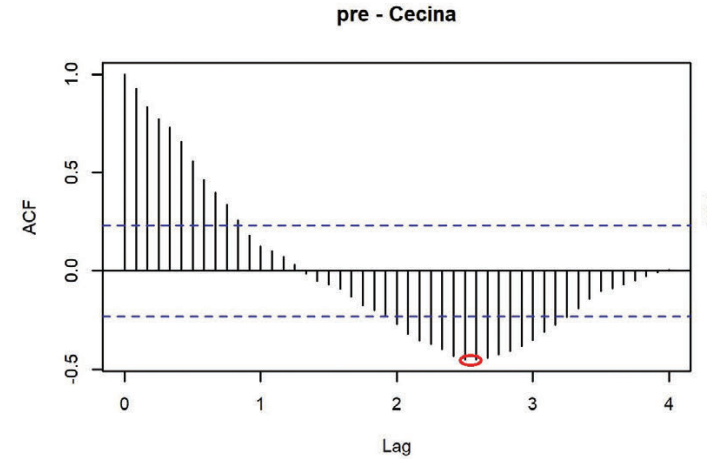

I diagrammi per le serie più complete del pluviometro di Cecina e freatimetro $5 \mathrm{~A}$ risultano molto simili e sembrano indicare cicli pluriennali con semiperiodo intorno a 2,5 anni (periodo 5 anni).

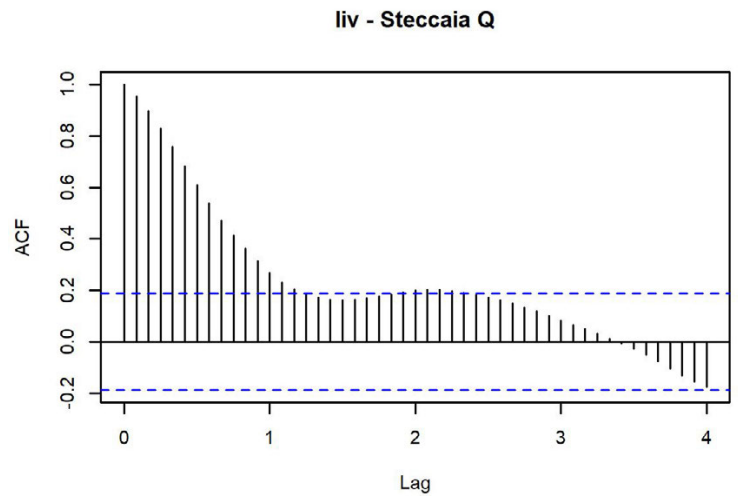

Fig. 7 - Autocorrelogram bydrometer Steccaia (liv).

Fig. 7 - Autocorrelogramma idrometro Steccaia (liv).

Risultati meno evidenti e non significativi si ottengono invece, sulla base dei dati disponibili, per la serie dell'idrometro di Steccaia.

Lo studio di correlogrammi derivati da serie temporali è analogo allo studio dell'autocorrelazione, con la differenza che la traslazione ripetuta di un definito lag avviene tra una prima ed una seconda variabile. I lags indicati nella Figura 8 corrispondono ancora a intervalli mensili con l'unità pari ad un anno e le linee tratteggiate indicano anche qui le soglie di significatività statistica. Un massimo di CCF che supera la soglia di significatività indica un lag significativo. Un lag negativo significativo indica un arretramento della prima stazione rispetto alla seconda.

Sono state scelte le due stazioni più rappresentative: pluviometro di Cecina e stazione freatimetrica $5 \mathrm{~A}$.

In questo caso la Figura 8 mostra una quantificazione del ritardo atteso per la freatimetria. Le oscillazioni delle precipitazioni al pluviometro Cecina precedono (lag negativo) quelle del livello freatimetrico di 5A di circa quattro mesi.

La stessa tecnica di decomposizione è stata applicata alla serie temporale dell'immagazzinamento netto cumulato, ottenendo la separazione dell'idrogramma nelle tre distinte componenti: trend, seasonal e random. Le tre componenti rappresentano distinti volumi delle riserve idriche sotterranee modellate nel periodo che, in accordo con Celico (1986), liv - 5A

Fig. 6 - Cecina rain gauge (pre) and 5 A groundwater level (liv) autocorrelograms.

Fig. 6 - Autocorrelogrammi pluviometro Cecina (pre) e freatimetro 5A (liv).

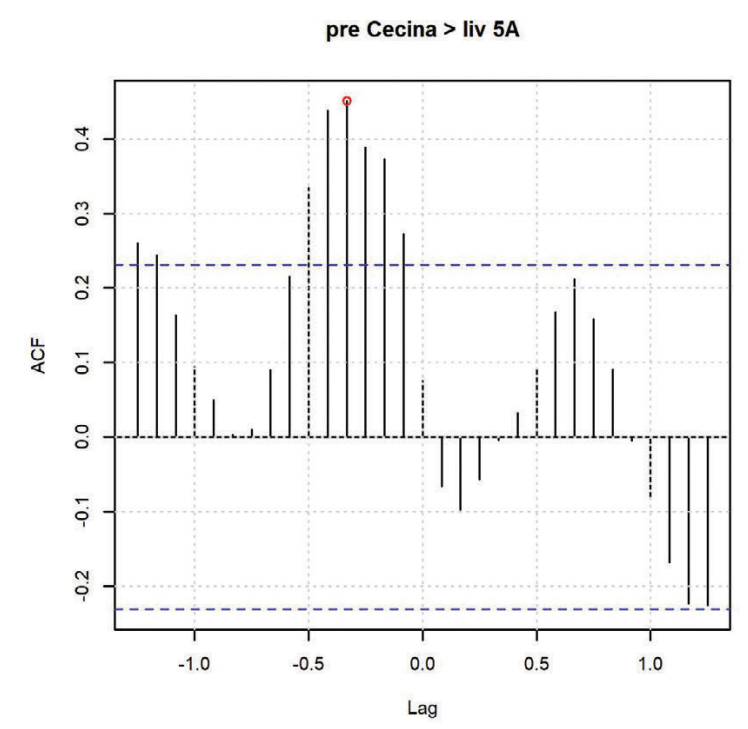

Fig. 8 - Crosscorrelogram Cecina rain gange versus phreatimeter $5 \mathrm{~A}$.

Fig. 8 - Crosscorrelogramma pluviometro Cecina rispetto al freatimetro 5A.

possono essere distinte in:

- $\quad$ permanenti (o geologiche)-volumi d'acqua immagazzinati al di sotto della minima quota di sfioro che non possono venire a giorno in modo naturale, ma solo per captazione;

- regolatrici - volumi immagazzinati tra il livello delle riserve permanenti e il livello piezometrico alla fine dell'esaurimento, resi disponibili solo in occasione di eventi di crisi idrica eccezionale;

- dinamiche - volumi d'acqua annualmente immagazzinati tra i livelli piezometrici ad inizio e fine del periodo d'esaurimento.

La decomposizione dell'idrogramma dell'immagazzinamento cumulato può permettere dunque di riferire una stima:

- della componente stagionale all'escursione media delle riserve dinamiche;

- della componente trend, all'escursione sul periodo delle riserve regolatrici anche secondo eventuali cicli e periodicità pluriennali;

- della componente random residuale a perturbazioni casuali, in taluni casi anche severe come nella magra autunnale 2012 .

Con riferimento alla Figura 9 le escursioni delle prime due componenti quantificano dunque in $2,5 \mathrm{Mm}^{3}$ annui la riserva dinamica e $22 \mathrm{Mm}^{3}$ quella regolatrice. 
Decomposition of additive time series

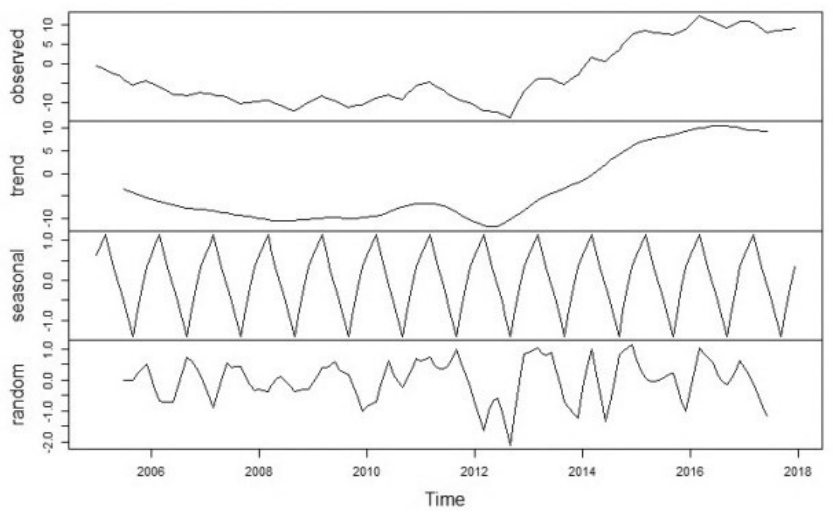

Fig. 9 - Time series decomposition of the cumulative net storage.

Fig. 9 - Decomposizione della serie temporale immagazzinamento netto cumulato .

Per quanto riguarda la ciclicità l'analisi di autocorrelazione condotta sulla variabile dell'immagazzinamento netto cumulato (Fig. 10) riporta un primo minimo relativo corrispondente ad un semiperiodo per 5 anni ed un secondo più profondo minimo assoluto corrispondente ad un semiperiodo di 7,5 anni circa.

Se è dunque disattesa una precisa corrispondenza con la ciclicità già notata per afflussi ed escursioni piezometriche (semiperiodo di 2,5 anni, periodo di 5 anni) appare tuttavia singolare come i semiperiodi di 5 e 7,5 anni, riscontrati per le ciclicità della funzione serbatoio, rappresentino comunque multipli interi (doppio e triplo), ipotetici armonici, del segnale originario delle precipitazioni.

Il confronto dei volumi idrici Wd e Wr con i volumi di prelievo Q, compresi tra 4,8 ed 8,8 $\mathrm{Mm}^{3}$ per una media di $6,4 \mathrm{Mm}^{3}$ nel periodo simulato dal modello, evidenzia come i prelievi superino attualmente le riserve dinamiche annuali $(\mathrm{Q}>\mathrm{Wd})$ con un ruolo attivo, operato di conseguenza dalla

\section{S - cumulative net storage}

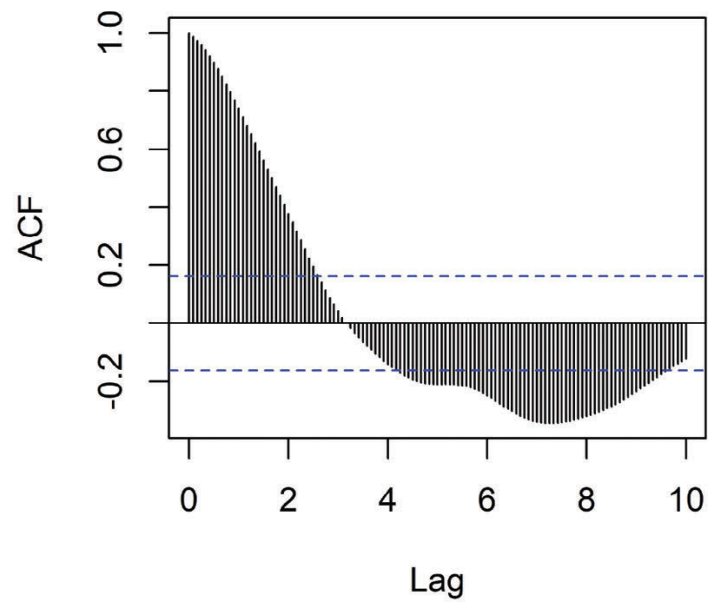

Fig. 10 - Autocorrelation of the cumulative storage series.

Fig. 10 - Autocorrelazione della serie dell'immagazzinamento cumulato. riserva regolatrice. Gli stessi volumi medi annui di prelievo compresi entro le oscillazioni della riserva regolatrice $(\mathrm{Q}<\mathrm{Wr})$ sembrano tuttavia garantire, su cicli pluriennali, il riassorbimento di deficit temporanei. Il tempo di esaurimento $(\mathrm{Wr} / \mathrm{Q})$ è compreso al massimo in cinque anni.

Lo stesso bilancio analitico, come nell'esempio riportato in Figura 11, è ripetibile su porzioni discrete del sistema acquifero come i corpi idrici già identificati come $\mathrm{A}, \mathrm{B}$ ed $\mathrm{M}$, corrispondenti a precisi ambiti di gestione della risorsa dell'autorità regolatoria.

L'idrogramma del corpo idrico A ben evidenzia come, a fronte di una maggiore capacità di immagazzinamento, il corpo idrico risulti maggiormente dipendente dalla ricarica. In presenza di eventi critici, quali il 2012, ha infatti mostrato una forte escursione con un minimo molto pronunciato.

Il corpo idrico $\mathrm{M}$, di spessori e dimensioni minori, ha una capacità di immagazzinamento più limitata, ma la sua diretta connessione con il fiume Cecina, sempre che si mantengano deflussi
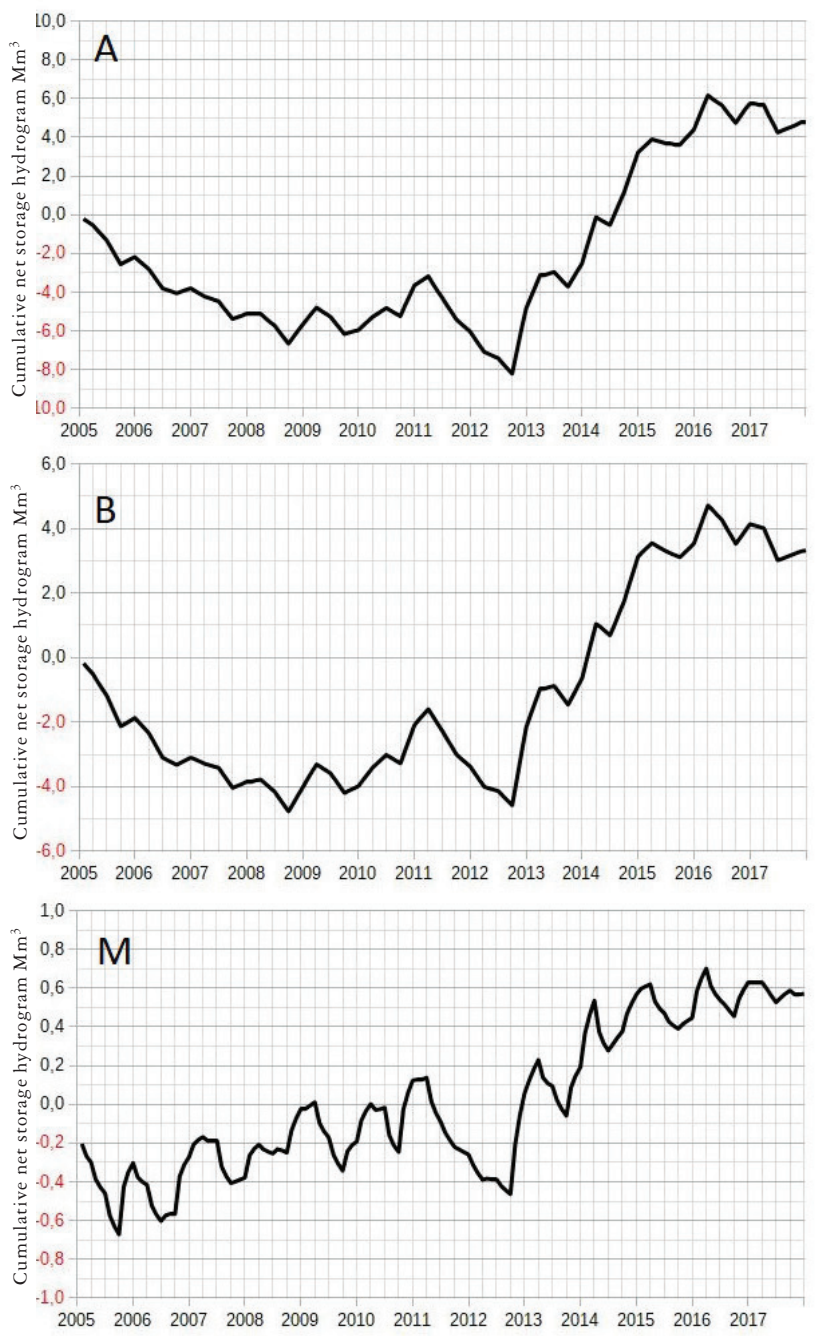

Fig. 11 - Cumulative net storage (IMC) bydrograms for water bodies A, B and $M$.

Fig. 11 - Idrogrammi dell'immagazzinamento netto cumulato (IMC) per i corpi idrici A, B ed M. 
sufficienti in questo tratto terminale, ha permesso negli anni una relativa costanza, se non incremento dei livelli idrometrici.

Il corpo idrico B ha, infine, caratteristiche intermedie tra A e M, mostrando una discreta capacità di immagazzinamento e una connessione, seppure indiretta, con il fiume Cecina, che ha permesso anche qui, nel 2012, di non raggiungere il minimo idrico assoluto.

\section{Conclusioni}

Tecniche di analisi delle serie temporali, in particolare decomposizione e derivazione $\mathrm{di}$ autocorrelogrammi e correlogrammi, sono state applicate alle variabili in ingresso ed uscita di una modellazione numerica di flusso in regime transitorio realizzata nel 2018 da ARPAT sulla Pianura del Cecina (LI).

Le serie storiche analizzate si riferiscono da un lato a misure dirette su variabili idrologiche (quali precipitazioni e livelli idrometrici) e, dall'altro, agli esiti della modellazione come gli stessi livelli piezometrici ed il bilancio analitico, quest'ultimo sintetizzato nella serie temporale dell'immagazzinamento netto cumulato.

Lanalisi delle serie temporali qui descritta è stata riferita ai punti di misura maggiormente significativi, in quanto direttamente influenti nel modello numerico (ARPAT 2018). In futuro, con un ulteriore implementazione del modello sia in senso temporale, sia di maggior dettaglio delle condizioni al contorno e di calibrazione, sarà possibile estendere ed approfondire l'analisi qui proposta ad un maggior numero di punti.

Oltre alla decomposizione delle serie temporali si è proceduto nell'analisi dell'autocorrelazione e cross correlazione, identificando una possibile ciclicità con semiperiodo di 2,5 anni ed un preciso tempo di risposta, valutato in quattro mesi circa tra precipitazioni locali ed escursioni piezometriche. Le stesse analisi condotte sull'idrogramma dell'immagazzinamento netto cumulato, che rappresenta la misura dei volumi invasati nel corpo idrico, restituiscono periodi maggiori, ma curiosamente multipli interi per 5 e 7,5 anni di semiperiodo. Si tratta di un processo che, sebbene non compreso fino in fondo, ha forse relazione con l'inevitabile e attesa trasformazione di un segnale più intermittente, quale è la precipitazione meteorica, in un segnale derivato più continuo e di maggiore durata.

I risultati ottenuti sono proposti come valido supporto alla gestione del bilancio idrico del corpo idrico costiero. Le componenti separate tramite l'analisi dell'immagazzinamento sono state ricondotte a due significative componenti del bilancio idrico: la riserva regolatrice $\mathrm{Wr}\left(22 \mathrm{Mm}^{3}\right)$ e la risorsa dinamica Wd $\left(2,5 \mathrm{Mm}^{3}\right)$. Dal confronto di queste componenti con i volumi di prelievo medio annuo da pozzi $\left(6,4 \mathrm{Mm}^{3}\right.$ nel periodo 2005 -2017) si ottiene una misura evidente e robusta, in quanto derivata da un'analisi di lungo periodo, di un bilancio idrico potenzialmente critico, vulnerabile a periodi con ripetute tendenze climatiche avverse. E' in questi casi che la modellazione operativa, unita all'analisi delle serie temporali prodotte, può rappresentare un utile strumento per valutare azioni preventive correttive e di pianificazione.
Acknowledgements

Thanks to the Regional Hydrological and Geological Service for the data provided and to Andrea Chahoud of ARPA ER for the precious technical information.

\section{Funding}

The work derives from a re-elaboration of the "Hydrogeological modeling for the management of water resources" report published at the conclusion of the non-mandatory institutional activities (INO), carried out by ARPAT for the Tuscany Region by Executive Decree of RT No. 9987 of 5/2/2017.

\section{Competing interest}

The authors declare no competing interest.

Author contributions

Conceptualization, SM and ST; methodology, SM and ST; writingoriginal draft preparation, SM and ST; writing-review and editing, SM and ST; All authors have read and agreed to the published version of the manuscript.

Additional information

Supplementary information is available for this paper at https://doi.org/10.7343/as-2021-531

Reprint and permission information are available writing to acquesotterranee@anipapozzi.it

Publisher's note Associazione Acque Sotterranee remains neutral with regard to jurisdictional claims in published maps and institutional affiliations. 


\section{BIBLIOGRAFIA}

Ambrosio M, Fagioli MT (2001) Modellazione e simulazione numerica in idrogeologia: esempio di applicazione alla pianura costiera di Cecina. "Modeling and numerical simulation in bydrogeology: example of application to the coastal plain of Cecina". Acque Sotterranee - Italian Journal of Groundwater, n 70 - Aprile 2001

Armellini R, Baldini E, Del Seppia D, Franceschini F, Gori N, Menichetti S, Tessitore S (2015) Modello numerico di flusso e trasporto del sistema acquifero della pianura del Cecina (Toscana, Italia), con l'uso di elaborazioni GIS. "Groundwater Flow and Transport Model in Cecina Plain (Tuscany, Italy) using GIS processing" Acque Sotterranee - Italian Journal of Groundwater - AS12056: 045 $-054$

ARPAE (2011) Collaborazione tra Arpa e Agenzia Regionale di Protezione Civile per l'aggiornamento periodico del modello di flusso delle acque sotterranee della Conoide del Fiume Marecchia a supporto della previsione e gestione delle emergenze idriche. "Collaboration between Arpa and the Regional Civil Protection Agency for the periodic updating of the groundwater flow model of the Marecchia River Conoide to support the forecast and management of water emergencies", Bologna, gennaio 2011.

ARPAT (2003) Studio idrogeologico con utilizzo di modelli numerici di simulazione per la definizione dei meccanismi d'arricchimento in nitrati delle acque sotterranee nell'area compresa tra gli stradoni del Lupo, del Tripesce, la SS. $n^{\circ}$ 206, Vada e S. Pietro in Palazzi. "Hydrogeological study using numerical simulation models for the definition of the nitrate enrichment mechanisms of the groundwater in the area between the Lupo, Tripesce and SS roads. 206, Vada and S. Pietro in Palazzi". Relazione finale, gennaio 2003. http://www. provincia.livorno.it/fileadmin/Difesa_e_Protezione_del_Territorio/ Arpat_20Re lazione_20finale.pdf.

ARPAT (2018) Modellistica del Bacino del Cecina per la la tutela quantitativa l'ottimale gestione delle risorse idriche della Val di Cecina e nella fascia costiera livornese compresa tra Rosignano e Bibbona-Regione Toscana. "Cecina Basin modeling for the quantitative protection and optimal management of water resources in the Val di Cecina and in the Livorno coastal area between Rosignano and Bibbona - Tuscany Region" http://www.arpat.toscana.it/documentazione/catalogopubblicazioni-arpat/modellistica-idrogeologica-per-la-gestionedella-disponibilita-delle-risorse-idriche-pianura-di-cecina.

Baldini E, Benucci C, Menichetti S, Tessitore S, Viti ML (2004) Hydrogeological study using numerical model to explain nitrate enrichment in a south tuscany coastal area. $32 \mathrm{Nd}$ IGC International Geological Conference, Florence.
Bartoletti E, Mazzanti R, Squarci P (1985) Idrogeologia del territorio del Comune di Rosignano M.mo "Hydrogeology of the territory of the Municipality of Rosignano M.mo". Suppl. N 1 Quad. Mus. Stor. Nat. O 6: 247-283.

Brockwell PJ, Davis RA (1991) Time Series: Theory and Methods, $2^{\text {nd }}$ Edition, Springer Verlag, NY, pp. 373-375, 405-417.

Chen X, Wang X, Lian J (2021) Applicability Study of Hydrological Period Identification Methods:Application to Huayuankou and Lijin in the Yellow River Basin, China. Water 2021, 13, 1265. https:/doi.org/10.3390/w13091265

Genovese NA (2003) Modellizzazione Numerica dell'Intrusione Salina nell'Acquifero Costiero del Fiume Cecina. "Numerical Modeling of the Saline Intrusion in the Coastal Aquifer of the Cecina River" Tesi di Laurea in Ingegneria per l'Ambiente e Territorio l'Università la Sapienza di Roma.

Harbaugh AW, McDonald MG (1996) User's documentation for MODFLOW-96 an update to the U.S. Geological Survey modular finite difference groundwater model U.S. Geol. Surv. Open- File Rep, 96-485.

Hasda R, Md Ferozur R, Chowdhury SJ, Khademul IM, Quamrul HA (2021) Climatic data analysis for groundwater level simulation in drought prone Barind Tract, Bangladesh: Modelling approach using artificial neural network. Groundwater for Sustainable Development 10 (2020) 100361

Kendall M, Stuart A (1983) The Advanced Theory of Statistics, Vol.3, Griffin. pp.410-414.

Lee M, You Y, Kim S, Kim KT, Kim HS (2018) Decomposition of Water Level Time Series of a Tidal River into Tide, Wave and Rainfall-Runoff Components. Water 2018, 10, 14.

Meggiorin M, Passadore G, Bertoldo S, Sottani A, Rinaldo A (2021) Assessing the long-term sustainability of the groundwater resources in the Bacchiglione basin (Veneto, Italy) with the Mann-Kendall test: suggestions for higher reliability. Acque Sotterranee - Italian Journal of Groundwater, 10(1). https://doi.org/10.7343/as-2021-499

Polemio M, Dragone V (1999) Serie storiche piezometriche delle unità idrogeologiche pugliesi: regime piezornetrico, effetti climatici ed antropici. "Piezometric historical series of Apulian hydrogeological units: piezometric regime, climatic and anthropic effects." Atti del $3^{\circ}$ convegno nazionale sulla protezione e gestione dele acque sotterranee per il III millennio. Parma 13-14-15 ottobre 1999. Pubblicazione CNR GNDCI 2000 .

Rumbaugh JO, Rumbaugh DB (2007) Groundwater Vistas (Version 5.16): software published by Environmental Simulations Inc., Reinholds, Pennsylvania.

Venables WN, Ripley BD (2002) Modern Applied Statistics with S Fourth Edition. Springer-Verlag. 